\title{
The Influence of Interfacial Chemistry on Bonding During High-velocity Impact of Microparticles
}

Xi Chen ${ }^{1}$, Ahmed Tiamiyu ${ }^{1}$, Christopher Schuh ${ }^{1}$ and James LeBeau ${ }^{2}$

${ }^{1}$ Massachusetts Institute of Technology, United States, ${ }^{2}$ Massachusetts Institute of Technology, Cambridge, Massachusetts, United States

Cold gas dynamic spraying, often referred to as cold spraying (CS), has become an essential process to produce surface coatings. During this process, powder particles are deposited in the solid-state at high velocities onto a substrate to form a coating. CS uses kinetic rather than thermal energy for deposition so that undesired oxidation and chemical reactions are avoided to enable complex material designs with intermixing of different materials or to repair surfaces [1]. Successful permanent particle adhesion occurs above a critical velocity [2], where various phenomena can occur, including extremely localized deformation accompanied by adiabatic heating [3], development of mechanical interlocking at the interface [4], and the interaction of strong pressure waves with the expanding edge of the deforming particle [5]. For most metals, there is a broad understanding that the most effective CS bonding requires a breakdown of the native oxide layers on the surface of the particle and substrate to permit metal-on-metal contact and bond formation [6]. However, detailed studies of the chemistry of these oxides and their role on metallurgical bonding is scarce, and we therefore aim to provide a site-specific comprehensive analysis of oxide chemistry across the particle-surface interface of permanently-adhered particles.

In this presentation, observations from a model $\mathrm{Cu}$ system to investigate a unit process of $\mathrm{CS}$ will be discussed. We conducted site-specific analyses of surface oxide chemistry for individual particle impacts, from particles of well-known sizes previously launched with a laser-induced particle impact tester (LIPIT) [7,8]. To prepare these samples for electron microscopy, lamella at the center of particle-substrate bonded sites for different impact velocities were extracted using a FEI Helios 660 Nanolab Dual-Beam FIB/SEM system. Combining STEM imaging and spectroscopy with high spatial resolution, we analyze the chemical components at the particle-substrate interface. As shown in Figure 1, the unimpacted particle is characterized by the presence of a surface oxide whose thickness varies from $10-50 \mathrm{~nm}$, while nearabsence of surface oxide is found on the mirror-polished substrate. Furthermore, the starting particle contains $\sim 65 \mathrm{~nm}$-sized spherical oxide particles that are buried in the particle itself, some of which are close to the particle surface. Using imaging and spectroscopy, we will discuss the role of both native surface and intraparticle oxides on particle-substrate interfacial bonding during impact, as shown in Figure 2. We will also discuss the existence of amorphous carbon at the interface, from the particle periphery to the south pole, and by extension, its possible role in bonding. Aided by the high spatial resolution and chemical analysis of STEM, we will highlight how nanoscale investigations of a unit process of CS provide new insights to improve bonding.
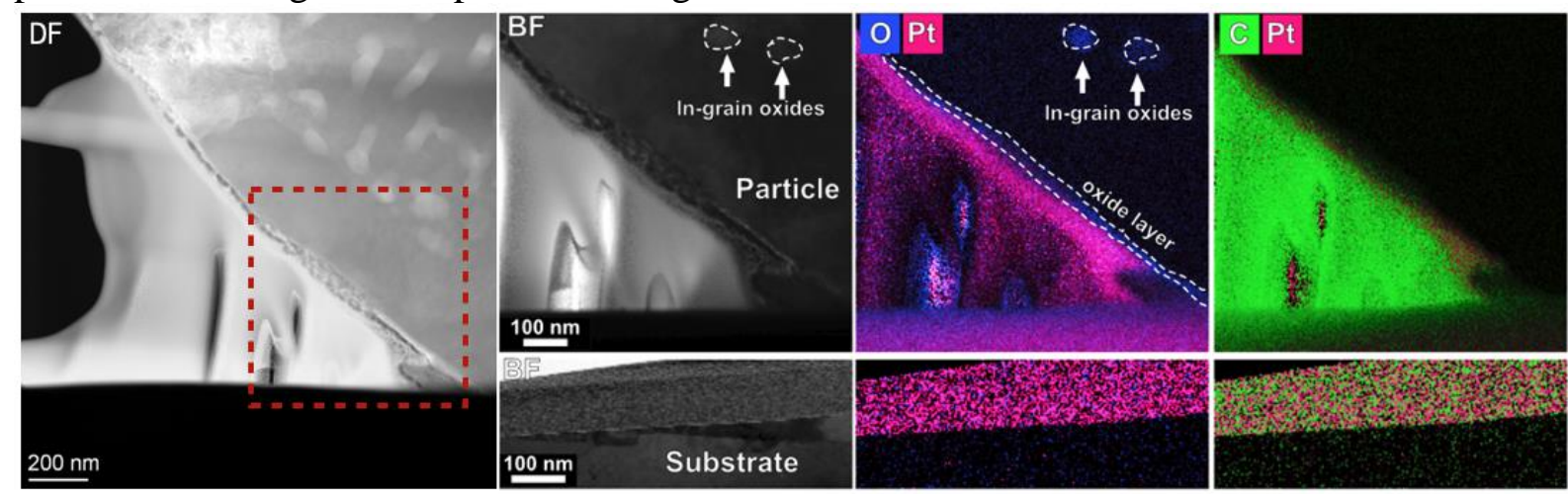
Figure 1. STEM chemical maps and images of the sample before impact. The surface of the particle and the substrate before impact is mapped with EDS with $\mathrm{O}$ given in blue, $\mathrm{Pt}$ in purple, and $\mathrm{C}$ in green.

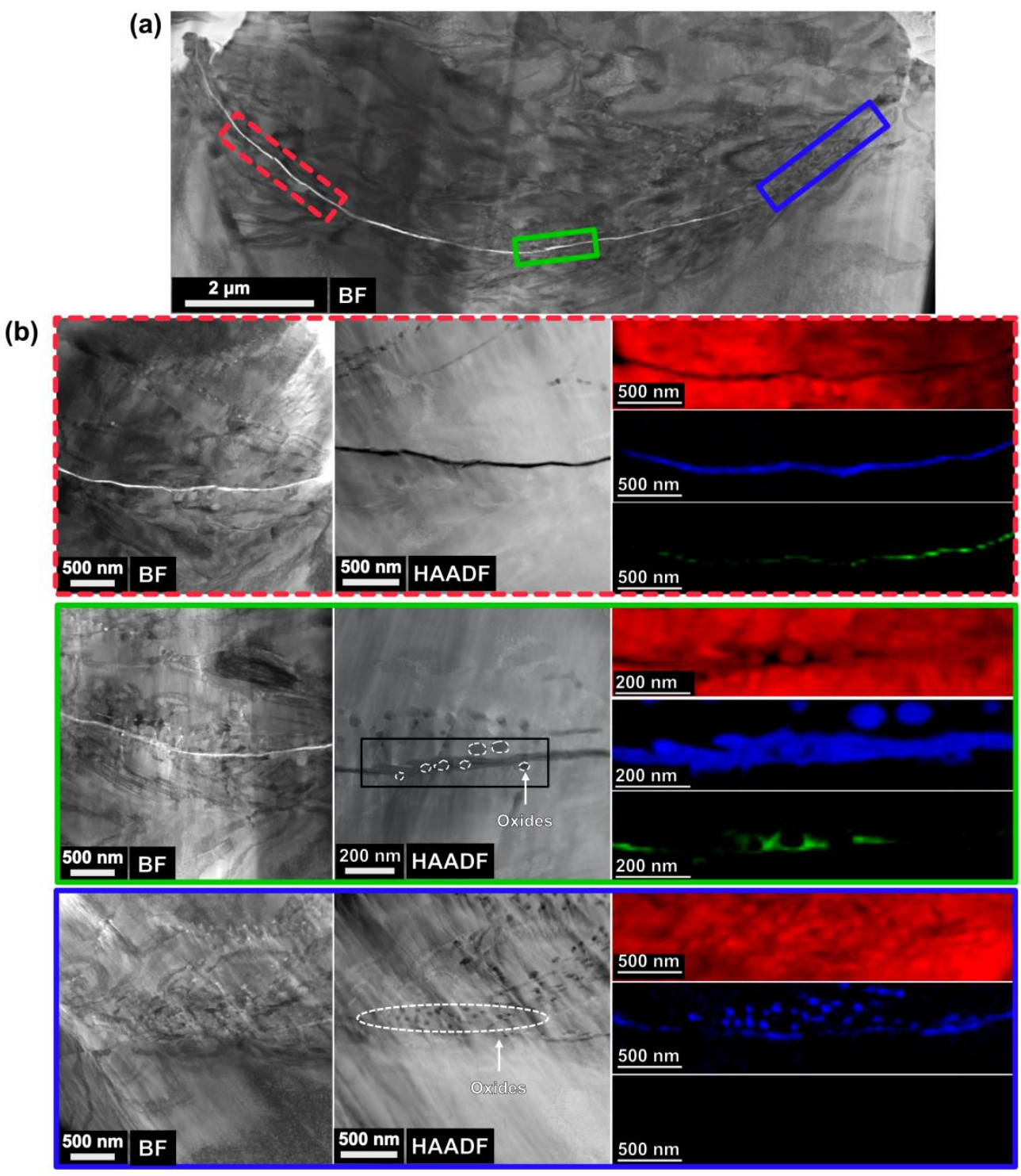

Figure 2. EELS analysis of the interface on different sides of sample $647 \mathrm{~m} / \mathrm{s}$. (a) Bright field STEM across impact interface and (b) BF and HAADF images, and EELS maps of the corresponding regions marked in (a) with $\mathrm{Cu}$ signal given in red, $\mathrm{O}$ in blue and $\mathrm{C}$ in green. Intraparticle oxides are marked in the HAADF images.

\section{References}

[1] Moridi, Atieh, et al. Surface Engineering 30.6 (2014), 369-395

[2] Grujicic, Mica, et al. Materials \& design 25.8 (2004), 681-688

[3] King, Peter C., et al. Journal of thermal spray technology 19.3 (2010), 620-634

[4] Hussain, Tanvir, et al. Journal of Thermal Spray Technology 18.3 (2009), 364-379

[5] Hassani-Gangaraj, Mostafa, et al. Acta Materialia 158 (2018), 430-439

[6] Assadi, Hamid, et al. Acta Materialia 116 (2016), 382-407

[7] Tiamiyu, Ahmed A., et al, Acta Materialia 202 (2021), 159-169 
[8] Tiamiyu, Ahmed A., Christopher A. Schuh, Surface and Coatings Technology 403 (2020),126386 [9] This work was performed with Thermo Fisher Scientific (TFS) Themis Z G3 at MIT nano. The support of U.S. Army Research Office under contract W911NF-13-D-0001 is appreciated. LIPIT experiments are supported by the U.S. Department of Energy, Office of Science, Office of Basic Energy Sciences, Division of Materials Sciences and Engineering under Award DE-SC0018091, And Y. Sun, D. Veysset and K. Nelson of MIT are acknowledged for their role in providing the LIPIT specimens in this work. AAT would like to thank the Natural Sciences and Engineering Research Council of Canada (NSERC) Postdoctoral Fellowship for financial support. 